\title{
Pathological changes in prostate lesions after androgen manipulation
}

\author{
R Montironi, C C Schulman
}

\begin{abstract}
The number of newly diagnosed cases of prostate cancer has doubled in the past four years because of the aging of the population coupled with growing awareness of the importance of early detection. The issues of clinical understaging and resection limit positivity have led to the development of novel management practices, including neoadjuvant hormonal treatment, which aims to downstage the primary tumour and decrease the positive margin rate before definitive localised treatment (radical prostatectomy or definitive radiation treatment (neoadjuvant)). There is conflicting evidence regarding pathological downstaging, with some studies suggesting benefit and others no benefit of androgen manipulation before radical prostatectomy. The problem might be related to incomplete sampling of the prostates and difficulties associated with the pathological interpretation of morphological changes. The least controversial aspect of neoadjuvant treatment is its impact on surgical margins. Most series have shown that neoadjuvant treatment in clinical $\mathrm{T} 2 \mathrm{tu}$ mours is associated with a $20-25 \%$ decrease in positive margins in radical prostatectomy specimens. In patients with clinical T3 tumours, the effects of neoadjuvant treatment on positive margins are less clear. Even if some early significant advantages can be observed following hormonal treatment this may not alter the metastatic spread and overall survival rate. Only long term follow up studies evaluating biological and clinical failures, time to progression, and survival will allow definitive conclusions from this approach.

(F Clin Pathol 1998;51:5-12)
\end{abstract}

Keywords: prostate cancer; androgens; neoadjuvant hormonal treatment; radical prostatectomy

The number of newly diagnosed cases of prostate cancer has doubled in the past four years. ${ }^{1}$ Factors that have contributed to this rise include the aging of the population coupled with growing awareness of the importance of early detection by measurement of serum prostate specific antigen (PSA) and digital rectal examination. Many of these prostate cancers, the most likely precursor of which is prostatic intraepithelial neoplasia (PIN), ${ }^{2}$ are curable. In the hope of improving long term disease free survival, urologists are now increasingly performing radical prostatectomy in men with prostate cancer that is localised on presentation who have a life expectancy of more than 10 years. ${ }^{3}$

During recent years, information has accumulated to suggest that the likelihood of finding organ confined untreated prostate cancer by pathological examination at the time of radical prostatectomy is only $50 \%$ in patients with clinically organ confined disease. ${ }^{13-5}$ In addition, tumour is present at the resection margin in approximately $10 \%$ to $20 \%$ of clinically defined $\mathrm{T} 1$ cases, $10 \%$ to $30 \%$ of $\mathrm{T} 2 \mathrm{a}$ cases, and $30 \%$ to $60 \%$ of $\mathrm{T} 2 \mathrm{~b}$ cases. $^{13-5}$ Patients with positive margins have a greater probability of local and distant recurrence than those with negative margins. Epstein et al found that margin positivity for tumours more strongly predicted disease free progression than did capsular penetration. ${ }^{67}$ The issue of clinical understaging and resection limit positivity have led to the development of novel management practices, including neoadjuvant hormonal treatment. The hypothesis surrounding the use of hormonal treatment before either radical prostatectomy or definitive radiation treatment (neoadjuvant) suggests that systemic hormonal treatment may be able to downstage the primary tumour and decrease the positive margin rate before definitive localised treatment - that is, to kill sufficient cells that the tumour regresses completely, or involutes into the gland. ${ }^{8}$

Action of hormones on the prostate

A metabolite of testosterone, dihydrotestosterone (DHT), influences the growth of prostate tissue, including tumour tissue. Testosterone is synthesised primarily in the testes, in response to luteinising hormone releasing hormone (LHRH), originating in the hypothalamus. The hypothalamus signals the pituitary gland, which responds by secreting luteinising hormone $(\mathrm{LH})$ into the bloodstream. LH signals the testes to produce testosterone. Other androgens closely related to testosterone are 
produced in the adrenal glands. Endogenous circulating concentrations of androgens can feed back to the hypothalamus and pituitary to shut off the production of LHRH. ${ }^{9}$

DHT is synthesised from testosterone by the type 1 and type 2 isozymes of nuclear membrane bound $5 \alpha$ reductase. The type 1 isozyme is predominantly expressed in the skin and liver, locations in which type 2 is also present. Expression of $5 \alpha$ reductase type 2 isozyme in the prostate is detected in basal epithelial cells and stromal cells. Thus, most intraprostatic DHT synthesis is accomplished by the type 2 isozyme in these two types of human prostate cell. Once synthesised, DHT acts in a paracrine fashion on the androgen dependent luminal or secretory epithelium, a hypothesis consistent with the distribution of androgen receptors in the nuclei of luminal cells, to drive cell growth. In addition, DHT produced in the skin and liver can act in a true endocrine fashion on prostate cells. DHT acts on its target tissue by binding to a cytoplasmic receptor and stimulating protein production, which results in cell division and growth. DHT also appears to inhibit programmed cell death, and this may be its most important contribution to the growth of the tumour..$^{10}$

\section{Hormonal treatment of prostate cancer}

TOTAL ANDROGEN BLOCKADE

Any treatment that reduces the concentration of male hormones is called hormonal treatment. Some of these treatments use hormones or drugs that mimic hormones to interfere with the cycle of testosterone production; others do not use hormones at all. The early therapies used surgical castration (orchiectomy) or a female hormone, diethylstilbestrol (DES), to reduce the supply of testosterone to the prostate, or a combination of these treatments. ${ }^{9}$

LHRH analogues (compounds similar to the hormone released by the hypothalamus to begin the cycle of testosterone synthesis) have been shown to be as effective as castration or DES without the risk of serious cardiovascular side effects. LHRH analogues interfere with the delicately balanced chemical feedback system that stimulates and controls testosterone production in the testes. After causing a brief surge of testosterone synthesis owing to the superagonist action, their continued administration eventually results in a shutdown of the synthesis of the hormones from the hypothalamus and the pituitary that control production in the testes. The effect of administering these drugs mimics the surgical procedure of removing the testes. A new class of LHRH analogues currently in development are LHRH antagonists. These agents immediately shut off gonadotropin secretion because they are direct antagonists of the LHRH receptor. ${ }^{9}$

Antiandrogens, such as bicalutamide and flutamide, are compounds able to interfere with the interaction of DHT with its receptor in prostate cells. Antiandrogens preferentially occupy the receptor, displacing DHT and preventing it from stimulating the cells to grow and divide. However, feedback mechanisms signal a shortage of testosterone/DHT reach- ing the cells and result in increased production of testosterone, some of which will reach and occupy the receptor. ${ }^{9}$

As understanding of the hormonal system advanced, researchers began to look for other, potentially better, ways to interfere with supply of androgen to the prostate. Orchiectomy, DES and LHRH analogues all act to shut down the production of testosterone in the testes. However, as much as $10 \%$ to $20 \%$ of the body's supply of male hormones are now believed to be produced in the adrenal glands. These androgens, which are closely related to testosterone, can promote prostate cancer growth. Thus, the concept of total androgen blockade has been postulated to provide greater efficacy in decreasing the overall effects of androgens on prostate gland growth. This treatment practice uses a combination of an LHRH analogue with an antiandrogen to eliminate both sources of androgens. ${ }^{10}$

INHIBITION OF $5 \alpha$ REDUCTASE

DHT is converted from testosterone by the enzyme $5 \alpha$ reductase. The administration of a selective inhibitor of $5 \alpha$ reductase, such as finasteride for six months, is considered an effective treatment for some patients with benign prostatic hyperplasia (BPH), without causing severe androgen deficiency effects in other tissues. ${ }^{11}$ Data on the effect of finasteride on prostatic neoplasia indicate that long term treatment with this drug could have a different effect according to the type of lesion treated: treatment induced changes could be readily identified in low grade prostate cancer and PIN, but less so in areas of Gleason grade 4 or 5 tumour. ${ }^{12}$ However, the regressive changes are not as severe as those induced by total androgen ablation. Testosterone, even though not converted into DHT, remains in the prostatic tissue and exerts some affect on the cells.

BASIC EFFECTS OF NEOADJUVANT HORMONAL TREATMENT

Androgen deprivation blocks proliferative activity and induces cell involution followed by apoptosis in hormone sensitive epithelial secretory cells. In treated cases there are no mitotic figures whereas the nuclear immunoreactivity for proliferating cell nuclear antigen (PCNA) or Ki67 is lower than in untreated cases. The frequency of apoptotic cells is greater than in untreated patients and increases from normal prostate/BPH, through PIN, to prostate cancer. ${ }^{13}$

Androgen insensitive cells are present early in the evolution of prostate cancer and lack treatment induced morphological changes. After androgen ablation, these cells eventually proliferate and the tumour progresses to an androgen independent state. In particular, cells with neuroendocrine differentiation, which exert a paracrine influence on growth of surrounding cells, do not express androgen receptors. Increasing neuroendocrine differentiation has been observed in tumours that became resistant to hormone treatment. Small cell prostate cancer is 


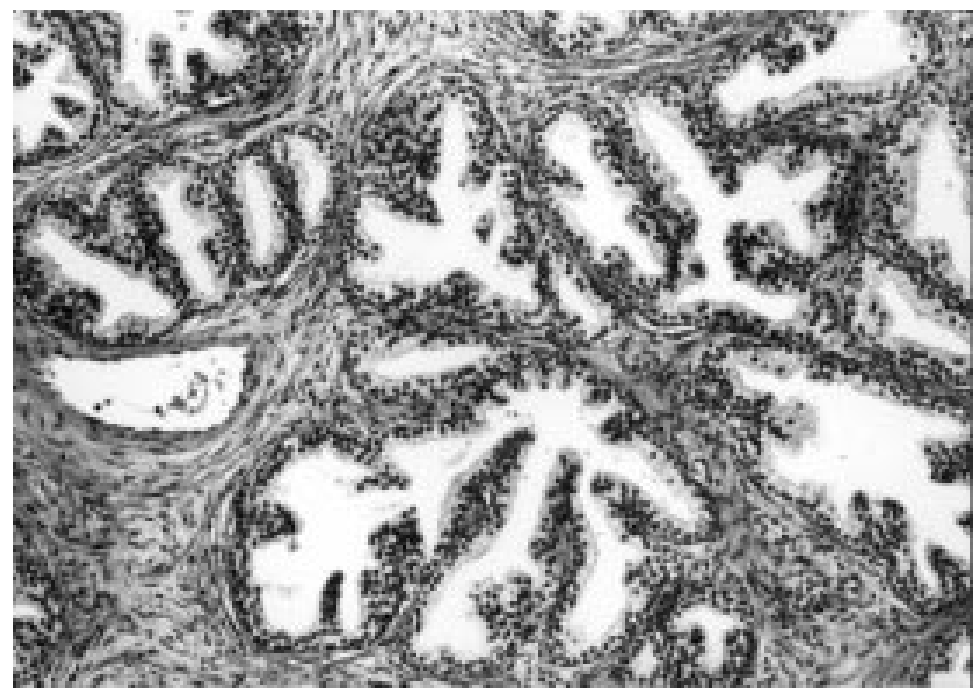

Figure 1 Untreated non-neoplastic prostate (transition zone). The ducts and acini have simple rounded contours that are not perfectly circular because of prominent undulations of the epithelial border. Both ducts and acini are lined by columnar secretory cells having pale cytoplasm and separated from the basement membrane by a flattened basal cell layer (original magnification $\times 160$ ).

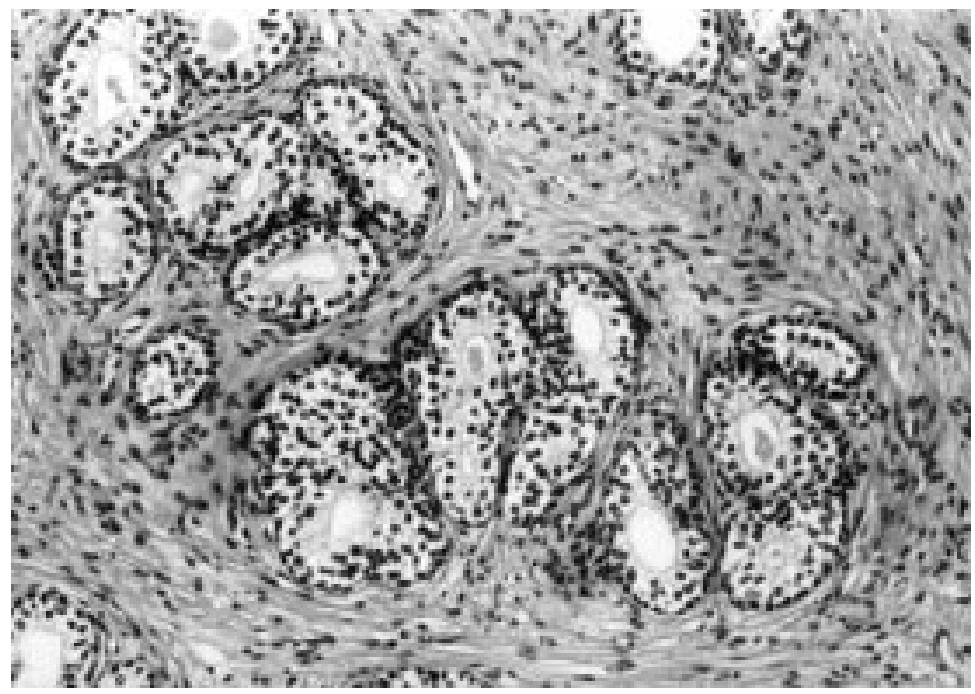

Figure 2 In contrast to untreated cases, the transition zone of treated non-neoplastic prostate shows simplification of the glandular lobules. The ducts and acini are small, ovoid, round or comma-shaped. A two cell-type epithelium is recognisable. The secretory cells have inconspicuous nucleoli, nuclear shrinkage, chromatin condensation, and cytoplasmic clearing. The basal cell layer is prominent (original magnification $\times 160$ ).

frequently neuroendocrine in differentiation and usually non-responsive to hormonal treatment. ${ }^{14}$

Whole mount sectioning of complete radical prostatectomy specimens is the best method for accurately evaluating the spectrum of morphological changes induced by total androgen blockade on benign, preinvasive, and malignant prostate lesions as well as the effect of androgen ablation on pathological stage and resection limit status of prostate cancer. In general the positive surgical margin rate is highly dependent on the technique the pathologist uses to process and evaluate the radical prostatectomy specimens. Positive margins are more likely identified when the prostate is totally embedded than when a random sampling technique is used. Pathologists need to be aware of the difficulties associated with the interpretation of specimens obtained after hormonal withdrawal, and should be extremely
Table 1 Pathological findings in the prostate following androgen blockade. The changes affect normal prostate, prostatic intraepithelial neoplasia, and cancer

Architecture

Prominent acinar atrophy with loss of hyperplastic glandular architecture in the transition zone

Basal cell prominence and hyperplasia in benign prostate Squamous metaplasia, including the immature variant

Decrease in extent of prostatic intraepithelial neoplasia

Decrease in extent of prostatic adenocarcinoma with loss of luminal (acinar) architecture

Decreased frequency of intraluminal crystalloids in prostatic adenocarcinoma

Cytology

Cytoplasmic clearing and vacuolisation

Nuclear shrinkage

Nuclear hyperchromasia

Nuclear pyknosis with increased frequency of apoptotic bodies Lack of mitotic figures

Loss of nucleolar prominence

Stroma

Focal hypercellularity

Focal lymphocytic/histiocytic infiltrate (including foamy, lipid laden histiocytes)

careful about possible misinterpretations of the morphological changes. Whenever in doubt, the pathologist should step section the specimen and use immunohistochemistry (see tissue immunohistochemistry of residual carcinoma). ${ }^{1516}$

DURATION OF NEOADJUVANT HORMONAL

TREATMENT

The optimal duration of neoadjuvant hormonal treatment is unknown. Most morphological data are from studies in which the investigators have arbitrarily used three months of continuous treatment with an LHRH analogue and an antiandrogen before radical prostatectomy. In other trials this treatment has been administered for six months or more. ${ }^{9}$ One of these is the Italian multicentric study (or PROSIT study) in which goserelin and bicalutamide were given for six months. Interim results indicate that the morphological changes are more pronounced than after three months of treatment. The concept of intermittent neoadjuvant hormonal treatment has recently gained popularity, based on experimental studies showing that a greater number of tumour cells are destroyed than with continuous treatment ${ }^{17}$; however, morphological data are not yet available.

\section{Morphological changes of prostate lesions} NON-NEOPLASTIC PROSTATE AND BENIGN PROSTATIC HYPERPLASIA

The overall appearance of hormonally treated prostates is that of global senescence or involution of the gland (figs 1 and 2). The transition zone shows simplification of the glandular lobules, although the lobular configuration is retained. The ducts and acini are small, ovoid, round or comma-shaped. There is no undulation of the epithelial border because of the presence of corrugations of the wall. The secretory cells have inconspicuous nucleoli, nuclear shrinkage, chromatin condensation, and cytoplasmic clearing. The basal cell layer is prominent and focal, immature, squamous metaplasia and basal cell hyperplasia are seen. Within the peripheral and central zones there is inconspicuous branching of the ducts and acini 


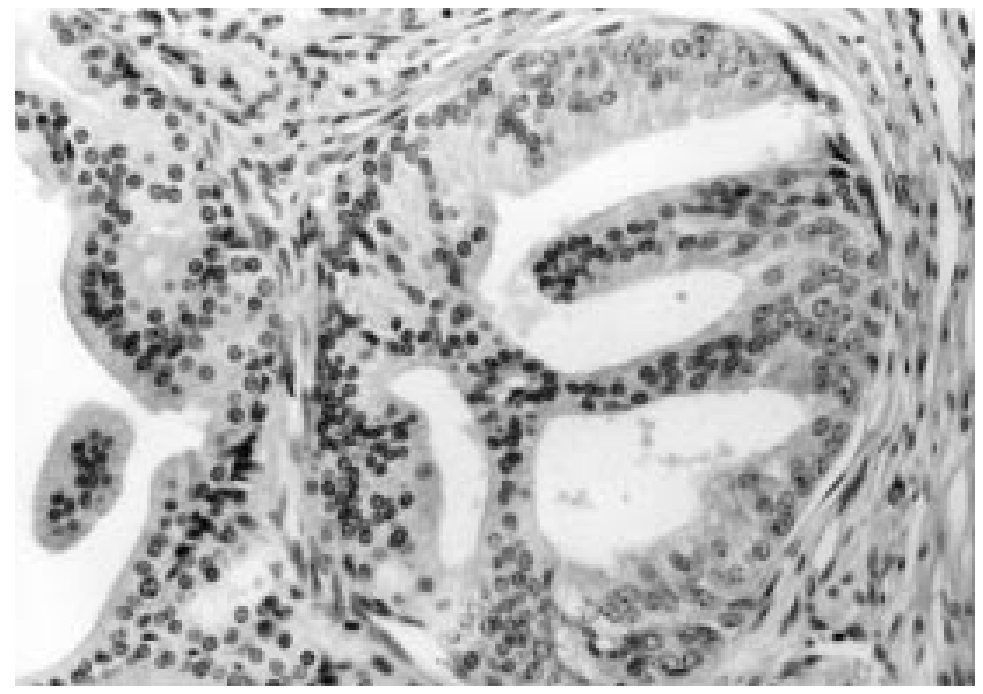

Figure 3 Untreated prostatic intraepithelial neoplasia with cribriform pattern (PIN of high grade). In contrast with untreated non-neoplastic transition zone (fig 1), cell crowding is present and accompanied by irregular nuclear spacing as well as an increase in nuclear size. In addition to the increased size variability, nuclei show finely granular chromatin and prominent nucleoli (original magnification $\times 250$ ).

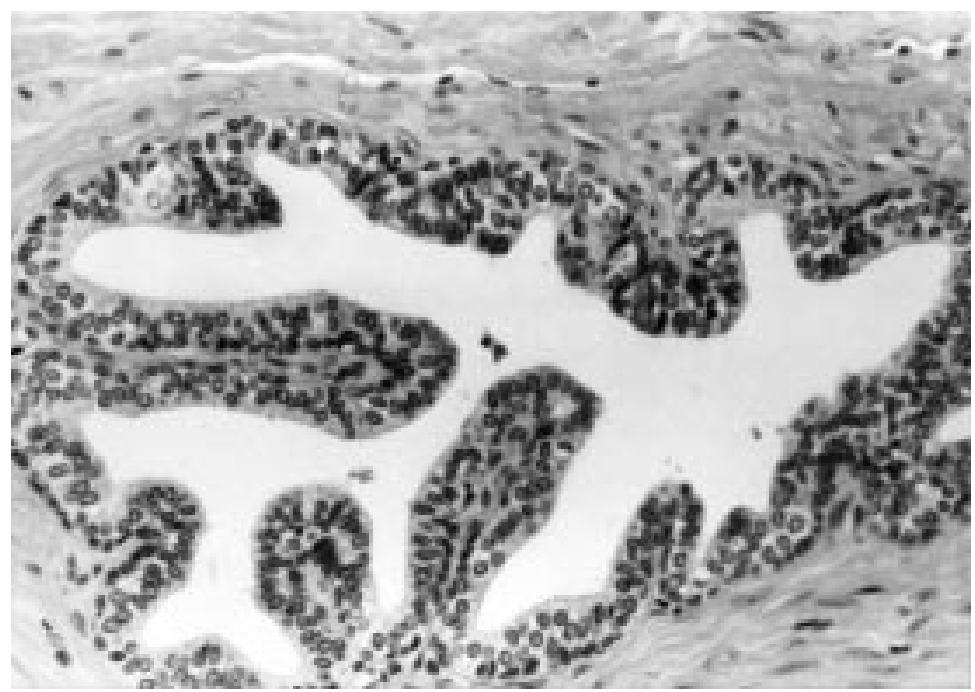

Figure 4 Treated prostatic intraepithelial neoplasia. The epithelial cell lining shows a recognisable basal cell layer. A certain degree of secretory cell-type stratification is present Crowding is less evident than in the untreated prostate. The lumen contains some cells, including apoptotic bodies (original magnification $\times 250$ ).

that appear dilated, star-shaped, and lined by flattened atrophic epithelium, which is usually single layered and seldom double layered (table 1)..$^{13}$

PROSTATIC INTRAEPITHELIAL NEOPLASIA

In treated patients, PIN is generally observed in the peripheral zone and is localised in scattered ducts. Ferguson and colleagues ${ }^{18}$ and Vaillancourt and colleagues ${ }^{19}$ demonstrated that the prevalence and extent of PIN is lower in treated glands. The cell lining shows a basal cell layer that is recognisable in most instances. A certain degree of secretory cell-type stratification is always present. However, crowding is less evident than in the untreated prostate. The cells have nuclear shrinkage, chromatin condensation, and pyknosis, inconspicuous nucleoli, and cytoplasmic clearing. Apoptotic cells are easily identifiable in all the cell-type layers. The duct and acinar lumen is always rich in cells: some are macrophages, some sloughed secretory cells with degenerative features, and others are apoptotic cells. No clear post-treatment grading of PIN is possible (figs 3 and 4$).^{20}$

\section{PROSTATIC ADENOCARCINOMA}

Hormonal treatment results in a significant overall reduction in the volume of prostate cancer compared with untreated prostate cancer in radical prostatectomy specimens from clinically confined disease. Vaillancourt et al reported a mean cancer volume of $4.66 \mathrm{ml}$ in the untreated group versus $2.11 \mathrm{ml}$ in the group of patients treated before surgery. ${ }^{19}$ However, calculating cancer volume in hormonally treated prostate is very difficult because residual disease is often present as scattered and multiple microscopic foci within a tissue section, which makes the calculation of tumour volume imprecise.

In general, histological response seems to correlate with the tumour pattern and the Gleason grades observed before androgen ablation treatment is started. Moreover, following total androgen ablation the morphological changes are more pronounced than after hormonal monotherapy - that is, LHRH analogue or antiandrogen used alone. Residual prostate cancer invading the prostatic capsule, periprostatic soft tissue, seminal vesicle, and metastatic to pelvic lymph nodes, shows treatment induced changes similar to adenocarcinomas confined within the prostate gland. ${ }^{19-28}$

Treated tumours with acinar pattern (primary Gleason grade 1 to 3 ) in the biopsy before androgen ablation treatment, show neoplastic acini that appear shrunken, a decreased frequency of intraluminal crystalloids, and areas of individual infiltrating tumour cells separated by abundant connective tissue (fig 5 and 6). The epithelial tumour cells have cytoplasmic clearing and enlargement by coalescence of vacuoles and rupture of cell membranes. The nuclear chromatin shows different changes, which range from a mild condensation - which barely allows the distinction between coarse chromatin granules (corresponding to heterochromatin) and finely dispersed chromatin (corresponding to euchromatin) - to a tightly condensed state similar to that observed in apoptosis. ${ }^{17}$ Similar to treated PIN, apoptotic bodies are easily identifiable in all epithelial cell layers as are macrophages and sloughed epithelial cells in the lumina. ${ }^{22}$ The hallmark of all untreated adenocarcinomas is that the tumour nuclei are frequently multinucleolated, the nucleoli being prominent (mean diameter $1.47 \mathrm{~mm}$ ), marginated, and with a perinuclear halo. In treated cases the nucleoli become inconspicuous, without margination, and have a decreased mean diameter of $1.09 \mu \mathrm{m}$, the nucleolar diameter being $<1.0 \mu \mathrm{m}$ in $20 \%$ of tumours. ${ }^{19}$ Treated tumours with pretreatment cribriform and solid/trabecular patterns (primary Gleason grade 4 and 5) show nuclear and cytoplasmic changes that appear less pronounced than in the acinar pattern. 


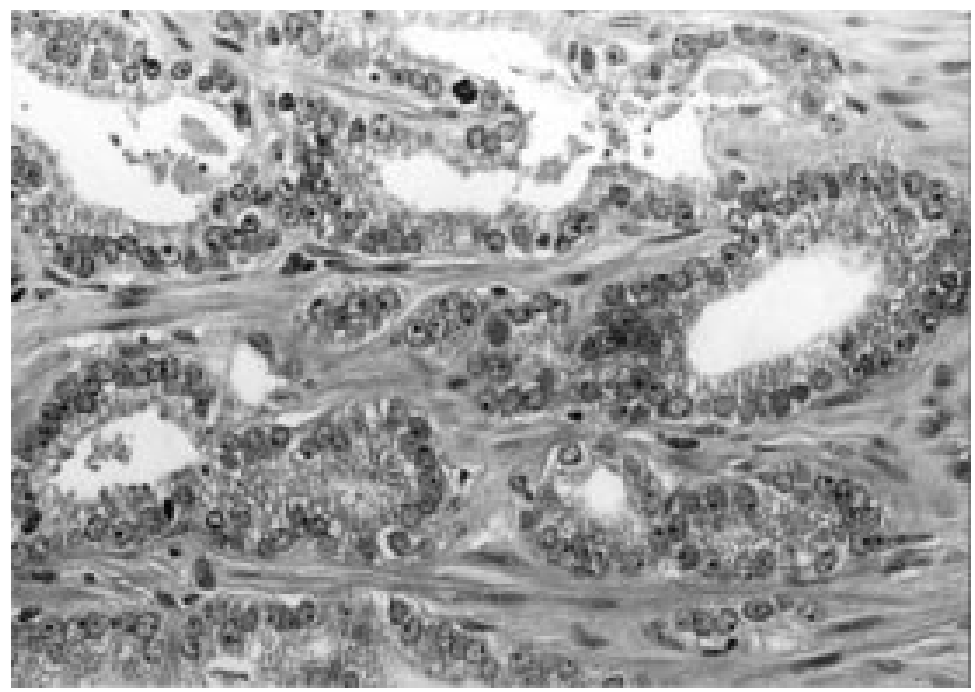

Figure 5 Untreated prostatic adenocarcinoma with acinar pattern. The hallmark is that the tumour nuclei are frequently multinucleolated, the nucleoli being prominent, marginated, and surrounded by a narrow clear space. The chromatin appears finely granular with some chromatin margination along the inner surface of the nuclear membrane. The cytoplasm of the small and large acinar patterns is moderately clear. The cell boundaries are recognisable (original magnification $\times 250$ ).

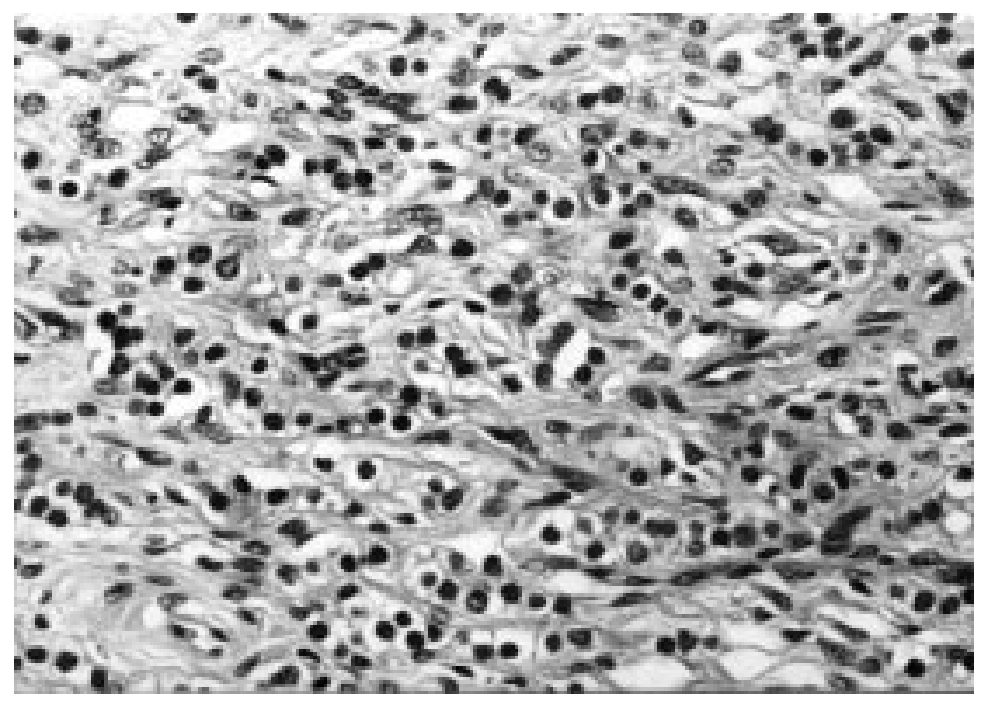

Figure 6 Treated prostatic adenocarcinoma. Compared with untreated cases, treated adenocarcinoma shows neoplastic acini that appear shrunken with areas of individual infiltrating tumour cells separated by abundant interglandular connective tissue. The epithelial tumour cells shows inconspicuous nucleoli, nuclear shrinkage, chromatin condensation, and cytoplasmic clearing and enlargement (original magnification $\times 250$ ).

The stroma shows variable degrees of fibrosis, reduced capillary vascularity, and variable density of lymphocytic infiltrates, often intermingled with mast cells, plasma cells, and eosinophils. Infiltrates of foamy histiocytes, difficult to distinguish from prostate cancer cells with clear cytoplasm, are sometimes present. ${ }^{28}$ Periprostatic fibrosis, obscuring the normal cleavage plane and making the operation more difficult, is reported after hormonal treatment. ${ }^{4}$ The longer patients receive hormonal treatment before surgery, the more fibrosis is observed around the prostate; there have not been any detailed qualitative and quantitative histological studies on the degree of fibrosis and its specific location. Based on a preliminary morphological evaluation, it appears that there is an increased thickness of the fibrous connective tissue septa that usually transverses the adipose tissue surrounding the capsule. Focal areas in which the fatty tissue is totally obliterated by connective tissue is sometimes present laterally, posteriorly, and around the seminal vesicles. It cannot be excluded that this feature represents tumour induced stroma in which cancer cells have regressed because of hormonal treatment.

\section{Tissue immunohistochemistry of residual} carcinoma

In some cases residual tumour may be very focal, consisting only of isolated cells or cell clusters within prostatic stroma. Identification of these tumour foci is made more difficult by the presence of cellular, tumour associated stroma and condensation of nuclear chromatin masking the prominent nucleoli of prostate cancer cells. Significant difficulty may be encountered in separating minute clusters and single file ribbons of tumour cells from lymphocytes, myocytes, and fibroblasts. Small foci of residual prostate cancer may also be missed in $4 \%$ to $15 \%$ of prostatectomy specimens. ${ }^{19}$ Immunostaining with PSA and prostatic acid phosphatase can detect persisting tumour cells, though reduction in the intensity of staining following hormonal treatment limits their usefulness. ${ }^{25}$ Cytokeratin immunohistochemistry may be necessary to confirm the presence of residual prostate cancer, especially for pathologists with limited experience in these cases. Immunostaining with a low molecular weight cytokeratin, such as CAM 5.2 antibody, will stain residual prostate cancer cells, but the same cells will lack reactivity for high molecular weight cytokeratin $343 \mathrm{E} 12 .{ }^{21}$ The latter is expressed only in the basal cells of the prostate and not in secretory acinar epithelium, whether benign, preinvasive or malignant. Gleave et al found that $50 \%$ of the cases that exhibited no residual cancer on routine pathological assessment had remaining foci of cancer discovered by immunostaining. ${ }^{9}$ Without the aid of additional step sections and immunostaining for cytokeratin these cases would have been reported as being stage pT0. ${ }^{15}$ The usefulness of immunohistochemistry was also shown by Gould et al in a study related to colloid pools and slit-like or haemangiopericytoma-like spaces devoid of lining cells occasionally present in the stroma of radical prostatectomy specimens subsequent to androgen deprivation treatment. ${ }^{29}$ These spaces showed strong immunostaining for A-80, an onco-developmental mucinous glycoprotein expressed only in preneoplastic and neoplastic prostatic cells, combined with sporadic cytokeratin reaction. The authors suggested that at least some of these spaces represent remnants of carcinomatous glands. ${ }^{29}$

\section{Pathological grading of treated adenocarcinoma}

A third of treated prostate cancer cases are classified as high grade (Gleason score 8 to 10 ), whereas, of the $56 \%$ that are of intermediate grade (Gleason score 5 to 7), most are Gleason score $7 . .^{18}{ }^{19}$ These percentages are much higher than expected in radical prostatectomy 
specimens-that is, there is a significant upgrading. Murphy's group ${ }^{24}$ considered it likely that a shift to a higher grade in the prostate was largely an artefact of treatment induced regression and caused by the destruction of most of the tumour bulk, the residual tumour often comprising numerous, isolated, single cells. These cells are non-viable and show pronounced treatment effects. According to others, higher grading could be explained by selective apoptosis of the more hormone sensitive (low grade) cells, leaving mainly higher grade tumour cells in the specimen, and leading to an increase in Gleason score. ${ }^{4}$ It has been argued that tumour upgrading is the result of selection of an androgen insensitive clone, more likely to be poorly differentiated. ${ }^{30}$ Data from the literature do not support this hypothesis.

Because of treatment induced morphological changes, grading of residual prostate cancer, based on standard Gleason criteria, is not accurate and is discouraged. ${ }^{25}$ Montironi et al developed a Bayesian belief network (BBN) for the identification of prostate cancer with hormone treatment changes from prostate cancer with poor to no treatment effect and from untreated prostate cancer. ${ }^{31}$ The BBN allowed identification with high certainty of prostate cancer with treatment related changes from those with either poor/no treatment effect or untreated. There was complete agreement between the network results and the clinical information of whether there was androgen deprivation before surgery. There was a statistically significant correlation between pretreatment tumour grade and network results. Montironi et al concluded that a BBN for the evaluation of androgen deprived prostate cancer offers a descriptive classification that is readily implemented in the evaluation of the degree of induced changes and allows the use of descriptive linguistic terms.

Van de Voorde et al devised a regressive score system in a study on radical prostatectomy specimens from patients with localised prostate cancer treated either with flutamide or estramustine phosphate (a hybrid molecule combining an oestrogen moiety and an alkylating agent).$^{28}$ The regressive score was based on the evaluation of nuclear pyknosis, cytoplasmic vacuolisation, degree of fibrosis, and interstitial lymphocytic infiltration. Each of these features had different outcomes expressed numerically. They found that the scores obtained from the patients treated with estramustine phosphate were higher than those from patients treated with flutamide. This system is similar to that published by Montironi et al. ${ }^{31}$ The main difference is that the network from the latter group gives the level of confidence on the regression grade.

\section{Effect of androgen ablation on pathological stage and resection limit status of prostate cancer}

Clinical downstaging has been mentioned in several studies but has not been confirmed in pathological examination in most cases. Moreover, reports of clinical downstaging must be interpreted with caution because approximately $25 \%$ of patients with clinical T3 tumour are overstaged. ${ }^{32}$

There is conflicting evidence regarding pathological downstaging, with some studies suggesting benefit and others no benefit of androgen manipulation before radical prostatectomy. In some studies, the problem might be related to incomplete sampling of the prostates and difficulties associated with the pathological interpretation of morphological changes. Interestingly, in the European multicentre prospective randomised study where the whole mount sectioning technique was adopted, pT2 tumours were statistically more common in the neoadjuvant treatment group $(48 \% v 24 \%$, $\mathrm{p}<0.01) .^{32}$ This seems to indicate that downstaging of clinical T2 tumours does occur with neoadjuvant treatment. In contrast, for clinical T3 tumours there was no significant difference between groups with respect to the final pathological stage. ${ }^{32}$ Trachtenberg ${ }^{33}$ pointed out that in most recent series there was no significant downstaging in patients with clinical T3 disease. These data support the concept that tumour cells massively invading the periprostatic tissue or the seminal vesicles do not return to the prostate after hormonal treatment.

Maximum downstaging - that is, complete disappearance of all neoplastic cells and the consequent complete absence of residual cancer in the specimen, has been reported anecdotally. However, systematic step sectioning of the specimen and immunohistochemistry have shown the presence of residual focal prostate cancer in specimens staged as pT $0 .^{29} 34$

A positive surgical margin should be defined as the presence of cancer at the inked margin of resection in a radical prostatectomy specimen. ${ }^{15}$ It indicates that tumour has not been completely excised. Positive margins may occur even in the absence of evident extracapsular disease. This is because of an inadvertent incision through the capsule into an organ confined cancer. Paulson ${ }^{35}$ reported that the status of the surgical margins was the most important prognostic feature in patients treated with radical prostatectomy.

The least controversial aspect of neoadjuvant treatment is its impact on surgical margins. ${ }^{36}$ Most series, whether prospective and controlled, and whatever the type of hormonal deprivation, have shown that neoadjuvant

Table 2 Influence of neoadjuvant hormonal treatment on positive surgical margins: results of recent prospective studies

\begin{tabular}{|c|c|c|}
\hline \multirow[b]{2}{*}{ Study } & \multicolumn{2}{|c|}{ Positive surgical margins (\%) } \\
\hline & $\begin{array}{l}\text { Radical } \\
\text { prostatectomy } \\
\text { only }\end{array}$ & $\begin{array}{l}\text { Neoadjuvant treatment } \\
\text { and radical } \\
\text { prostatectomy }\end{array}$ \\
\hline Fair et $a l^{\beta}$ & 33 & 10 \\
\hline Haggman et $a l^{41}$ & 58 & 31 \\
\hline Labrie et $a l^{40}$ & 38 & 13 \\
\hline Schulman et a $l^{30}$ & 57 & 32 \\
\hline Pedersen $e t a l^{42}$ & 46 & 24 \\
\hline Soloway et a ${ }^{28}$ & 47 & 17 \\
\hline Goldenberg et $a l^{43}$ & 65 & 28 \\
\hline Aus et $a l^{44}$ & 41 & 23 \\
\hline \multicolumn{3}{|c|}{ European multicentric study ${ }^{39}$} \\
\hline cT2 & 36 & 13 \\
\hline cT3 & 61 & 44 \\
\hline
\end{tabular}


treatment in clinical T2 tumours was associated with $20-25 \%$ decrease in positive margins in radical prostatectomy specimens. ${ }^{4}$ As an example, in the Memorial Sloan-Kettering Cancer Center study, the percentage of organ confined cancers in patients treated with immediate surgery was $49 \%$ whereas it was $77 \%$ in patients treated with neoadjuvant hormones. ${ }^{37}$ In patients with clinical T3 tumours, the effects of neoadjuvant treatment on positive margins are less clear (table 2)..$^{88^{39-44}}$ No difference was observed in the European prospective study. Neoadjuvant hormonal treatment does not alter the frequency of pelvic lymph node metastasis or seminal vesicle invasion. ${ }^{32}$

\section{Conclusions}

Short term neoadjuvant hormonal treatment before radical prostatectomy has been advocated by an increasing number of urologists. Most of them have shown downsizing of the prostate by some $30-50 \%$. Clinical downstaging has been demonstrated in about $30 \%$ but this could not be confirmed at the final pathological staging. Reduction of positive margins in patients receiving neoadjuvant treatment varies between $15 \%$ and $25 \%$ compared with control groups. Several biases may however complicate the analysis of the results, the main cause of misinterpretation being the difficulty encountered by the pathologist properly to process, evaluate, and grade the tumour after hormonal deprivation. Even if some early significant advantages can be observed, such as a decrease of positive margins, this may not necessarily alter the metastatic spread and overall survival rate. Only long term follow up studies evaluating biological (PSA) and clinical failures, time to progression, and survival will allow definitive conclusions from this approach. ${ }^{136} 45$

We acknowledge the helpful advice of Dr $\mathrm{H}$ Bharucha, Institute of Pathology, The Queen's University of Belfast, Belfast, UK.

1 Watson RB, Soloway MS. Neoadjuvant hormonal treatment before radical prostatectomy. Seminars Urol Oncol before radical prostat

2 Bostwick DG. Progression of prostatic intraepithelial neoplasia to early invasive adenocarcinoma. Eur Urol 1996; 30:145-52.

3 Schulman CC, Wildschutz T, Zlotta AR. Neoadjuvant hormonal treatment prior to radical prostatectomy: facts and questions. Eur Urol 1997;32 (suppl):41-7.

4 Bostwick DG, Myers RP, Oesterling JE. Staging of prostate cancer. Sem Surg Oncol 1994;10:60-72.

5 Catalona WJ, Smith DS, Ratliff TL, et al. Measurement of prostate-specific antigen in serum as a screening test for prostate cancer. N Engl f Med 1991;324:1156-61.

6 Epstein JI, Pizov G, Walsh PC. Correlation of pathological findings with progression after radical retropubic prostatefindings with progression after radi

7 Ohori M, Wheeler TM, Kattan MW, et al. Prognostic significance of positive surgical margins in radical prostatesignificance of positive surgical margins in radic
ctomy specimens. F Urol 1995;154:1818-24.

8 Fair WR, Aprikian A, Sogani P, et al. The role of neoadjuvant hormonal manipulation in localized prostatic cancer. Cancer 1993;71(suppl):1031-8

9 Gleave ME, Goldenberg SL, Jones EC, et al. Optimal duration of neoadjuvant androgen withdrawal therapy before radical prostatectomy in clinically confined prostate cancer. Sem Urol Oncol 1996;14(suppl 2):39-47.

10 Crawford ED, Eisenburger MA, McLeod DG, et al. A controlled trial of leuprolide with and without flutamide in prostatic carcinoma. $N$ Engl F Med 1989;321:419-24.

11 Montironi R, Diamanti L. Morphologic changes in benign prostatic hyperplasia following chronic treatment with a 5 - $\alpha$-reductase inhibitor finasteride. Comparison with combination endocrine therapy. F Urol Pathol 1996;4:123-35.

12 Civantos F, Soloway MS, Pinto JE. Histopathological effects of androgen deprivation in prostatic cancer. Sem Urol Oncol of androgen deprivation in

13 Montironi R, Magi Galluzzi C, Scarpelli M, et al. Quantitative characterisation of the frequency and location of cell proliferation and death in prostate pathology. $\mathcal{f}$ Cell Biochem 1994;19(suppl):238-45.

14 di Sant'Agnese PA. Neuroendocrine differentiation in prostatic carcinoma. Cancer 1995;75:1850-9.

15 Bazinet M, Zheng W, Begin LR, et al. Morphologic changes induced by neoadjuvant androgen ablation may result in underdetection of positive surgical margins and capsular involvement by prostatic adenocarcinoma. Urology 1997; 49:721-5.

16 Wojno KJ, Epstein JI. The utility of basal cell-specific anticytokeratin antibody ( 34 beta E 12) in the diagnosis of prostate cancer. A review of 228 cases. Am 7 Surg Pathol 1995;19:251-60.

17 Akakura K, Bruchovsky N, Goldenberg N, et al. Effects of intermittent androgen suppression on androgendependent tumors. Cancer 1993;71:2782-90.

18 Ferguson J, Zincke H, Ellison E, et al. Decrease of prostatic intraepithelial neoplasia (PIN) following androgen deprivation therapy in patients with stage $\mathrm{T} 3$ carcinoma treated by radical prostatectomy. Urology 1994;44:91-5.

19 Vaillancourt L, Tetu B, Fradet Y, et al. Effect of neoadjuvant endocrine therapy (combined androgen blockade) on normal prostate and prostatic carcinoma. I Surg Pathol 1996;20:86-93

20 Montironi R, Magi Galluzzi C, Muzzonigro G, et al. Effect of combination endocrine therapy on normal prostate, prostatic intraepithelial neoplasia and prostatic adenocarcinoma. F Clin Pathol 1994;47:906-13

21 Armas OA, Aprikian A, Melamed J, et al. Clinical and pathobiological effects of neoadjuvant total androgen ablation therapy in clinically localized prostatic carcinoma. $\mathrm{Am}$ f Surg Pathol 1994;18:979-91.

22 Magi Galluzzi C, Montironi R, Giannulis I, et al. Prostatic invasive adenocarcinoma. Effect of combination endocrine invasive adenocarcinoma. Effect of combination endocrine and location of proliferating cell nuclear antigen (PCNA). and location of proliferating cell nucle

23 Montironi R, Magi Galluzzi C, Fabris G. Apoptotic bodies in prostatic intraepithelial neoplasia and prostatic adenocarcinoma following total androgen ablation. Pathol Res Pract 1995;191:873-80.

24 Murphy WM, Soloway MS, Barrows GH. Pathologic changes associated with androgen deprivation therapy for prostate cancer. Cancer 1991;68:821-8.

25 Reuter VE. Pathological changes in benign and malignant prostatic tissue following androgen deprivation therapy. Urology 1997;49(suppl 3A):16-22

26 Smith DM, Murphy WM. Histologic changes in prostate carcinomas treated with leuprolide (luteinizing hormonereleasing hormone effect). Distinction from poor tumor differentiation. Cancer 1994;73:1472-7.

27 Têtu B, Srigley JR, Boivin J-C, et al. Effect of combination endocrine therapy (LHRH agonist and flutamide) on normal prostate and prostatic adenocarcinoma. Am f Surg Pathol 1991;15:111-20.

28 Van de Voorde WM, Elgamal AA, Van Poppel HP, et al. Morphologic and immunohistochemical changes in prostate cancer after preoperative hormonal therapy. Cancer 1994;74:3164-75.

29 Gould VE, Doljanskaia V, Gooch GT, et al. Stability of the glycoprotein A-80 in prostatic carcinoma subsequent to androgen deprivation therapy. Am F Surg Pathol 1997;21: 319-26.

30 Schulman CC, Sassine AM. Neoadjuvant hormonal deprivation before radical prostatectomy. Eur Urol 1993;24:450-5.

31 Montironi R, Bartels PH, Thompson D, et al. Androgendeprived prostate adenocarcinoma: evaluation of deprived prostate adenocarcinoma: evaluation of effect with a Bayesian belief network. Eur Urol 1996;30: effect with

32 Schulman CC, Witjes WPJ, Zlotta AR, et al, for the European Study Group on Neoadjuvant Treatment of Prostate Cancer. Neoadjuvant combined androgen deprivation therapy in locally confined prostate carcinoma: short-term results of a European randomized study. Molecular Urology. [In press.]

33 Trachtenberg J. Neoadjuvant androgen ablation therapy prior to radical prostatectomy: quantum leap or hope unrealised? Curr Opin Urol 1996;6:254-7.

34 Gleave ME, Goldenberg SL, Jones EC, et al. Biochemical and pathological effects of 8 months of neoadjuvant androgen withdrawal therapy before radical prostatectomy in patients with clinical confined prostate cancer. F Urol 1995; 155:213-19.

35 Paulson DF. Impact of radical prostatectomy in the management of clinically localized disease. $\mathcal{F}$ Urol 1994; management

36 Abbas F, Scardino PT. Why neoadjuvant androgen deprivation prior to radical prostatectomy is unnecessary. Urol Clin North Am 1996;23:587-604

37 Fair WR, Cookson MS, Stroumbakis N, et al. The indications rationale and results of neoadjuvant androgen deprivation in the treatment of prostatic cancer. Urology 1997;49(suppl):46-55.

38 Soloway MS, Sharifi R, Wood D, et al. Randomized comparison of radical prostatectomy alone or preceded by androgen deprivation for cT2 prostate cancer. $\mathcal{F}$ Urol 1995; 154:424-8.

39 Witjes W, Schulman CC, Forster G, et al, European Study Group on Neoadjuvant Treatment [abstract]. Eur Urol 1996;30(suppl 2):210.

40 Labrie F, Cusan L, Gomez JL, et al. Downstaging of early stage prostate cancer before radical prostatectomy: the first randomized trial of neoadjuvant combination therapy with 
flutamide and a luteinizing hormone-releasing agonist. Urology 1994;44:29-37.

41 Haggman M, Hellstrom M, Aus G, et al. Neoadjuvant GnRH agonist treatment (tryptorelin and cyproterone acetate) for flare protection and total prostatectomy. Eu Urol 1993;24:456-60.

42 Swedish POPCOM Group, Pedersen KV, Lundberg S, Hugosson $\mathrm{J}$, et al. Neoadjuvant treatment with triptorelin versus no treatment prior to radical prostatectomy: prospective randomized multicenter study [abstract]. $\mathcal{F}$ Urol 1995;153(suppl):391.
43 Goldenberg SL, Klotz LH, Jewett MAS, et al, The Canadian Urologic Oncology Group. Randomized, controlled study of neoadjuvant reversible androgen withdrawal therapy for prostate cancer. F Urol 1996;156:873-7.

44 Aus G, Abrahamsson PA, Goran A, et al. Pretreatment with triptorelin before radical prostatectomy - a 2 year followup. Eur Urol 1996;30A(suppl 2):209.

45 Schulman CC. Neoadjuvant androgen blockade prior to prostatectomy: a retrospective study and critical review. Prostate 1994;5(suppl):9-13 